\title{
Hypocapnia and cerebral ischaemia in hypotensive newborn piglets
}

\author{
A Whitelaw, B R Karlsson, K Haaland, I Dahlin, P A Steen, M Thoresen
}

\begin{abstract}
This study tested the hypothesis that hypocapnia superimposed upon hypotension produces a further reduction in cerebral blood flow velocity (CBFV). In 12 newborn piglets, CBFV was measured continuously by Doppler ultrasound through an artificial fontanelle. Hypotension was induced by removing $30 \mathrm{ml}$ $\mathrm{kg}$ of blood. Increasing the ventilator rate reduced the average arterial carbon dioxide tension from 5.5 to $2.0 \mathrm{kPa}$. When mean arterial pressure (MAP) was held steady at 45 $\mathrm{mm} \mathrm{Hg}$ or above, hypocapnia produced a substantial drop in CBFV but, in all the piglets with MAP below $38 \mathrm{~mm} \mathrm{Hg}$, hypocapnia failed to change CBFV by $10 \%$. Hypocapnia produced an increase in lactate in sagittal sinus blood but cerebral venous hypoxanthine concentrations were not affected by hypocapnia. Hyperventilation (without haemorrhage) produced a significant drop in MAP, preventable by infusing colloid.

Hypocapnia itself does not further reduce CBFV in the hypotensive piglet. However, the pressure effect of hyperventilation may significantly impair the cerebral circulation.
\end{abstract}

Despite the great improvements in the survival of premature infants, there are reports of increasing numbers of handicapped survivors with cerebral palsy. ${ }^{12}$ The most important neuropathological lesion associated with spastic diplegia in premature infants is periventricular leucomalacia. This is a bilateral cerebral lesion in the white matter furthest from the arterial blood supply. The aetiology is not fully understood and no treatment has been shown to be effective in preventing periventricular leucomalacia. On theorectical grounds, it is likely that hypotension is an important causative factor but the occurrence of periventricular leucomalacia is not consistently associated with hypotension. ${ }^{3}$ There must be other aetiological factors.

It has been proposed that a very low arterial carbon dioxide tension $\left(\mathrm{PaCO}_{2}\right)$ (hypocapnia) may be important in the aetiology of ischaemic brain damage in newborns. ${ }^{4}$ When blood pressure is normal, raising $\mathrm{PaCO}_{2}$ increases cerebral blood flow and decreasing $\mathrm{PaCO}_{2}$ decreases cerebral blood flow. When blood pressure falls, the brain tries to maintain blood flow by progressively vasodilating and eventually becomes maximally vasodilated. If hypocapnia caused cerebral vasoconstriction in this situation, cerebral blood flow could be reduced to a critically low level with ischaemic damage following. Although brain lesions have not been demonstrated in experimental animals after hypocapnia, ${ }^{5}$ slowing of the electroencephalogram has been noted in adult humans during hyperventilation. These changes could be reversed by hyperventilating with $100 \%$ oxygen. ${ }^{6}$ The normal escape mechanisms that inhibit excessive vasoconstriction may not operate in the immature neonatal cerebral circulation.

Inadvertent hypocapnia may occur when premature infants are mechanically ventilated for transport by ambulance or helicopter from the place of birth to the neonatal intensive care unit as infants do not usually have any form of carbon dioxide monitoring during transport. Hypocapnia may occur any time that an infant is mechanically ventilated without careful monitoring of $\mathrm{PaCO}_{2}$, for example during surgical anaesthesia. This is particularly likely with infants who do not have respiratory distress syndrome and stiff lungs.

Calvert et al reported a case-control study of 15 infants with periventricular leucomalacia. ${ }^{7}$ The infants with periventricular leucomalacia had experienced significantly lower $\mathrm{PaCO}_{2}$ values during the first 72 hours than had the control infants. The length of time that $\mathrm{PaCO}_{2}$ was below $3.3 \mathrm{kPa}$ was longer in the infants who subsequently developed periventricular leucomalacia. Calvert et al had no data on blood pressure but discussed the likelihood that the infants with periventricular leucomalacia had suffered hypotension as well as hypocapnia. Evidence for the involvement of hypocapnia and hypoperfusion in the aetiology of neurological deficit after cardiopulmonary bypass in adult patients has also been reported. ${ }^{8}$

In this study we tested the hypothesis that hypocapnia superimposed upon hypotension in the newborn can further reduce cerebral blood flow velocity and thereby increase the risk of cerebral ischaemia. None of the previous papers on hypocapnia, hypotension, and cerebral blood flow have dealt with newborns and the study would be unethical in human infants. The newborn piglet was chosen as the experimental model because of the great similarities in cardiovascular and respiratory systems, cerebral circulation, and body size between the piglet and human infant.

\section{Methods}

The experiments were performed on 12 newborn piglets aged from 12 hours to 3.5 days and weighing 1.1 to $2.8 \mathrm{~kg}$, which were kept with the sow until three hours before the

0514 Oslo 5

Accepted 2 April 1991 
experiment started. Venous access was secured during brief ether anaesthesia. An initial dose of $50 \mathrm{mg} / \mathrm{kg}$ of chloralose and $250 \mathrm{mg} / \mathrm{kg}$ urethane was given during 15 minutes and followed by a continuous infusion of $12.5 \mathrm{mg} / \mathrm{kg} /$ hour chloralose and $62 \mathrm{mg} / \mathrm{kg} /$ hour of urethane throughout the experiment. Maintenance fluid as $5 \%$ dextrose was given at $7 \cdot 5 \mathrm{ml} / \mathrm{kg} /$ hour. A rectal temperature of $39^{\circ} \mathrm{C}$ was maintained by a heating pad and overhead heater.

A tracheostomy was performed and each piglet was paralysed with pancuronium $0.2 \mathrm{mg} /$ $\mathrm{kg}$ intravenously and mechanically ventilated with $25-30 \%$ oxygen during surgery, care being taken to avoid any hypoxaemia. Both umbilical arteries and the umbilical vein were catheterised in piglets less than 48 hours old. In slightly older piglets a femoral artery and subclavian vein were catheterised. These catheters were used for blood sampling, continuous arterial pressure recording, and infusion of fluids and drugs. In five animals a catheter was inserted into the right internal jugular vein with the tip just below the brain and in two animals a catheter was inserted into the sagittal sinus. These catheters were used for hypoxanthine and lactate blood sampling. Blood samples were separated within one minute and plasma kept frozen until hypoxanthine was measured by high precision liquid chromatography. ${ }^{9}$ Plasma lactate was measured by standard laboratory techniques. In order to measure intracerebral blood velocities with Doppler ultrasound, an artificial fontanelle of $1 \mathrm{~cm}$ diameter was made just lateral to the midline where the cranial sutures meet.

All values are given as median values with the $95 \%$ confidence interval ${ }^{10}$ and are listed in the table. Comparisons of grouped data were made using the Wilcoxon signed rank test. ${ }^{10}$

The experimental protocol was as follows:

\section{HYPOCAPNIA DURING NORMOTENSION}

Median mean arterial pressure (MAP) at the start of the protocol was $48 \mathrm{~mm} \mathrm{Hg}$ (37 to 75 ). After recording cerebral blood flow velocity (CBFV) and MAP continuously for five minutes with a normal $\mathrm{PaCO}_{2}(5 \cdot 5 \mathrm{kPa})$, hyperventilation was produced by increasing the frequency of the ventilator from 20 (inspiratory time 0.3 seconds, expiratory time $2 \cdot 7$ seconds) to 100 breaths/ minute (bpm) (inspiratory time 0.3 seconds, expiratory time 0.3 seconds). This produced a rapid fall in $\mathrm{PaCO}_{2}$ within 10 minutes to $2 \cdot 0 \mathrm{kPa}$. Three animals received $20 \mathrm{ml} / \mathrm{kg} 5 \%$ albumin during hyperventilation to correct the tendency for MAP to fall. CBFV recording was continued while hyperventilation was continued for a further 10 minutes and then normal ventilation was resumed (18 to $20 \mathrm{bpm}$ ). The $\mathrm{PaCO}_{2}$ rose back to the normal range.

\section{HYPOCAPNIA DURING HAEMORRHAGIC} HYPOTENSION

With the $\mathrm{PaCO}_{2}$ at $5.4 \mathrm{kPa}$, blood was withdrawn through a catheter into heparinised syringes at a rate of $1-2 \mathrm{ml} / \mathrm{kg} / \mathrm{minute}$ until $30 \mathrm{ml} / \mathrm{kg}$ had been removed. This produced a median fall in
MAP of $37 \%$ to $32 \mathrm{~mm} \mathrm{Hg}$ as well as a median fall of $35 \%$ in CBFV.

\section{HYPOTENSIVE HYPOCAPNIA}

In a stable hypotensive condition, at least 30 minutes after the end of the bleed, baseline recordings with a normal $\mathrm{PaCO}_{2}$ were obtained. Hyperventilation was again produced by ventilating at $100 \mathrm{bpm}$. The $\mathrm{PaCO}_{2}$ fell from $5.5 \mathrm{kPa}$ to $1.9 \mathrm{kPa}$. Blood was transfused back into the piglet during hyperventilation at a rate sufficient to hold the MAP steady. Hyperventilation and recording of CBFV and MAP continued. After 20 minutes of hyperventilation the last blood samples were obtained and the experiment was terminated by rapid injection of potassium chloride.

\section{CBFV RECORDINGS}

As long as the cross sectional area of the vessel recorded from is constant, changes in blood velocity are proportional to changes in blood flow. ${ }^{11}$ A $10 \mathrm{MHz}$ Doppler ultrasound system (SD 100 Vingmed Sound) was used. By adjusting the depth of the sample volume and direction of insonication through the artificial fontanelle, precise localisation on an intracerebral artery could be achieved. In each piglet, the same artery $(1.7 \mathrm{~cm}$ deep and $1.5 \mathrm{~cm}$ lateral to the midline) was used for recording at a constant angle with the transducer held in place manually. The Doppler system was interfaced to an Apricot XI computer. Every $5 \mathrm{~ms}$ the computer was fed the mean CBFV calcualted from the Doppler spectrum, MAP, and heart rate. CBFV, $M A P$, and heart rate were all displayed in real time on a screen during the whole experimental period and stored on disc.

\section{Results}

HYPOCAPNIA DURING NORMOTENSION

Hyperventilating the piglets by increasing the ventilator rate from 20 to $100 \mathrm{bpm}$ produced an appreciable drop in MAP. This is shown in the table and illustrated in fig 1. MAP in nine piglets fell from a median of $48 \mathrm{~mm} \mathrm{Hg}$ (37-75) to a median of $36 \mathrm{~mm} \mathrm{Hg}$ (29-56) and in three piglets where albumin was given, MAP fell from a median of 46 to $41 \mathrm{~mm} \mathrm{Hg}$. Thus during hyperventilation the median MAP of all 12 piglets was $41 \mathrm{~mm} \mathrm{Hg} \mathrm{(33} \mathrm{to} 51$ ). In all the piglets with a MAP above $44 \mathrm{~mm} \mathrm{Hg}$ there was a definite fall in cerebral blood flow velocity during hyperventilation. These young piglets nearly all showed a proporational decrease in CBFV with MAP. Hence when calculating the effect of low $\mathrm{PaCO}_{2}$ on CBFV, the effect of blood pressure change has to be corrected for. This is illustrated in fig 1 where CBFV fell by nearly $50 \%$ whereas MAP fell by less than $25 \%$ during hyperventilation. Thus $25 \%$ was subtracted from the initial CBFV to calculate the starting value from which the change during hypocapnia was calculated. The table shows the CBFV fell by a median of $20 \%$ (10-33) during this normotensive hypocapnia. A cerebrovascular response to hypocapnia was considered to be present if CBFV fell by at least $10 \%$. 
Cardiovascular changes during experiment. Values are medians (95\% confidence intervals)

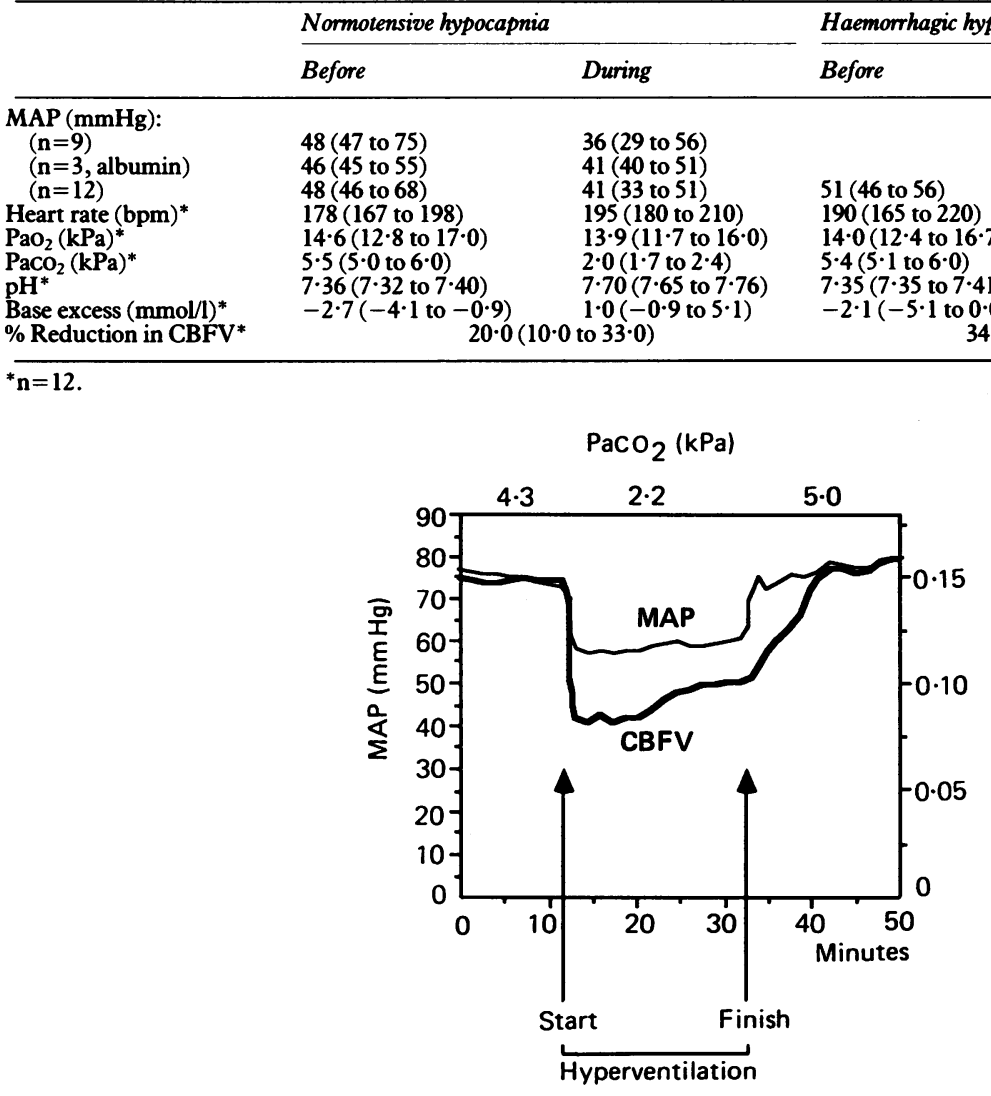

Figure 1 Continuous recording of $C B F V$ and $M A P$ in a piglet during hyperventilation with normal blood pressure. There is a fall in MAP but a much larger fall in CBFV indicating a cerebrovascular response to hypocapnia.

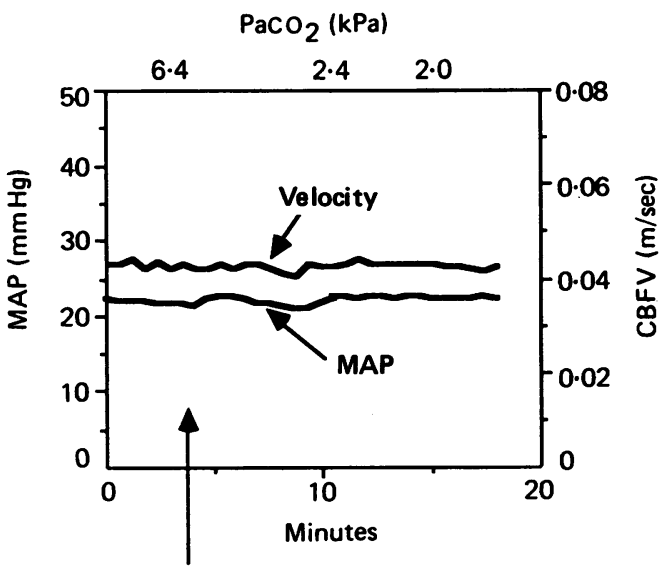

Start of hyperventilation

Figure 2 Continuous recording of $C B F V$ and $M A P$ in a piglet during hyperventilation with hypotension after haemorrhage. Blood was transfused back to maintain a steady blood pressure during hyperventilation. There was no change in $C B F V$ indicating an absent cerebrosvascular response to hypocapnia.

\section{HYPOCAPNIA DURING HAEMORRHAGHIC} HYPOTENSION

During hyperventilation after the haemorrhage, MAP was maintained by blood retransfusion. In eight out of nine piglets with MAP below 38 $\mathrm{mm} \mathrm{Hg}$, hypocapnia failed to produce any further fall in CBFV (fig 2), and the ninth piglet showed only a $6 \%$ fall. In one piglet with MAP

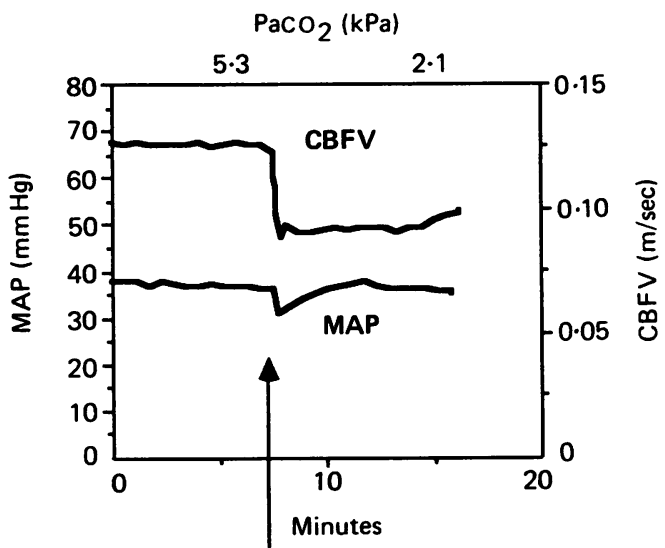

Start of hyperventilation

Figure 3 Continuous recording of $C B F V$ and $M A P$ during hyperventilation with $M A P$ at $38 \mathrm{~mm} \mathrm{Hg}$ after

haemorrhage. Albumin was transfused to maintain blood pressure. There was a large fall in CBFV indicating a cerebrosvascular response to hypocapnia.

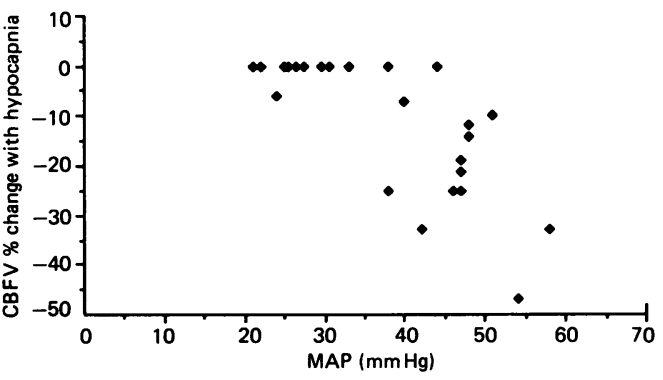

Figure 4 Percentage change in $C B F V$ in 12 piglets each with two hypocapnia challenges at differing blood pressures, before and after haemorrhage.

$38 \mathrm{~mm} \mathrm{Hg}$ after haemorrhage, the normal cerebrosvascular response to hypocapnia was preserved and this is illustrated in fig 3.

CEREBROSVASCULAR RESPONSE TO HYPOCAPNIA AT DIFFERENT MAP

When all 24 hyperventilation challenges are considered together as in fig 4 , it can be seen that the cerebrosvascular response to hypocapnia was clearly present when the MAP was over $44 \mathrm{~mm} \mathrm{Hg}$. When MAP was below $38 \mathrm{~mm}$ $\mathrm{Hg}, \mathrm{CBFV}$ responses were absent or less than $10 \%$.

Figure 5 shows that jugular venous or sagittal sinus plasma hypoxanthine concentration showed no significant increase during either normotensive hypocapnia or hypotensive hypocapnia. In the seven animals examined there was an increase in plasma hypoxanthine as the 


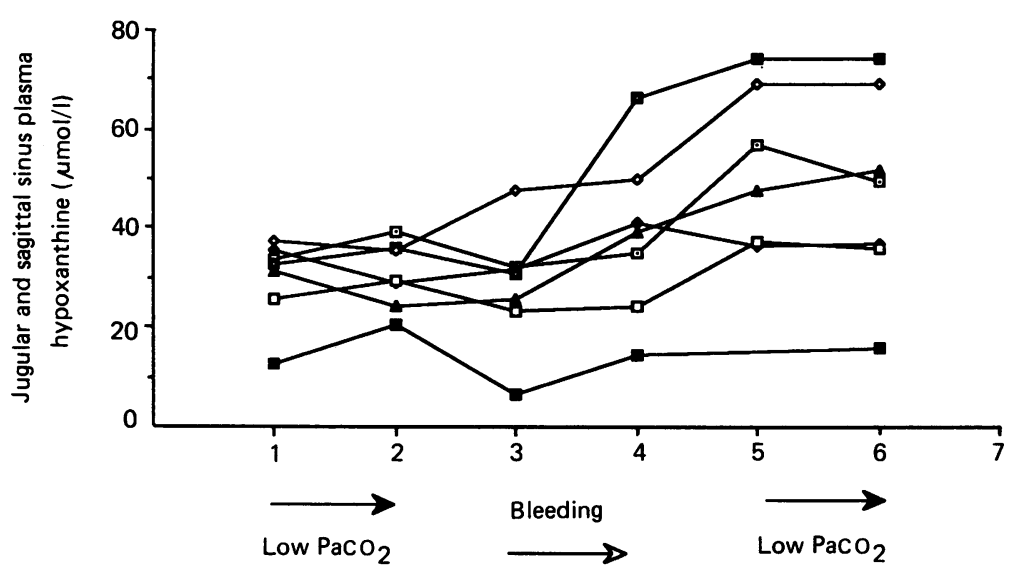

Figure 5 Serialplasmahypoxanthine concentrations in blooddraining the brain, either from the jugular vein (five piglets) or from the sagittal sinus (two piglets). Numbers on $X$ axis indicate samples before (1) and during (2) normotensive hypocapnia, before (3) and during (4) haemorrhagic hypotension and before (5) and during (6) hypotensive

hypocapnia. There was no change in plasma hypoxanthine during eithernormotensive $(2 v 1)$ or hypotensive (6v5) hypocapnia. There was a significant increase in hypoxathine after the haemorrhage $(5 v 3, p \leqslant 0.005)$

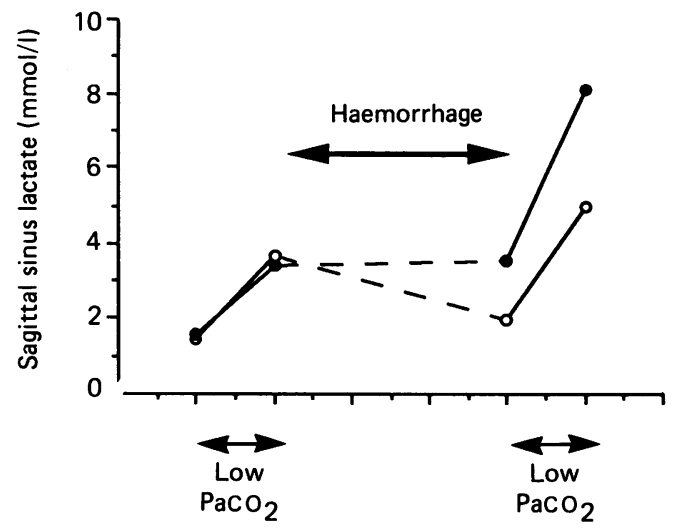

Figure 6 Plasma lactate concentration draining the brain via the sagittal sinus (two piglets). There was a definite increase in lactate during normotensive and hypotensive hypocapnia.

arterial pressure fell with blood loss. Figure 6 shows that sagittal sinus plasma lactate concentration increased during normotensive and hypotensive hypocapnia.

\section{Discussion}

When MAP was steady at a level below $38 \mathrm{~mm}$ $\mathrm{Hg}$, hypocapnia produced negligible reduction in CBFV. This is clear evidence against our hypothesis that hypocapnia could further impair the cerebral circulation during hypotension. It would be an appropriate physiological response for the cerebral circulation to vasodilate in response to hypotension and to try to maintain the best possible cerebral circulation at all costs. Thus it seems that the cerebral vasodilator response to hypotension cannot be over-ridden by the cerebral vasoconstrictor response to hypocapnia. Hypoxanthine is probably the best available plasma marker of cellular hypoxia (or failure to make ATP) ${ }^{12}$ and the lack of any significant increase in hypoxanthine in jugular or sagittal sinus blood is further evidence that hypocapnia does not, by itself, produce cerebral ischaemia.

The increase in lactate in sagittal sinus blood is not necessarily evidence of cerebral ischaemia. There is much evidence that the increase in $\mathrm{pH}$ with hyperventilation changes the activity of glycolytic pathway enzymes and thus increases lactate production while cerebral levels of phosphocreatine, ATP, and energy charge remain unchanged. ${ }^{13}$ Mitchenfelder and Theye have demonstrated in dogs that hypocapnia can shift the oxygen-haemoglobin dissociation curve in sagittal sinus blood to the left (Bohr effect) thus reducing oxygen availability to the tissues. ${ }^{15}$ This was associated with a change towards anaerobic metabolism. Lactate has been postulated to have a role as a cerebral vasodilator but the rise in lactate during hypotensive hypocapnia was not accompanied by further vasodilatation (fig 2). This finding suggests that the cerebral circulation was already maximally vasodilated during hypotension.

Do our findings mean that overventilation is harmless? Not necessarily. The anecdotal observations of an association between hypocapnia and subsequent neurological impairment may be due, not to a direct effect of hypocapnia on the cerebral circulation, but to the pressure effect of hyperventilation on the circulation. We observed that hyperventilation produced a median drop in MAP of $12 \mathrm{~mm} \mathrm{Hg}$. A fall in blood pressure of such magnitude combined with the Bohr effect on oxygen-haemoglobin dissociation could certainly impair cerebral oxygenation in a critically ill infant. We have previously described how increases in ventilator pressure can reduce CBFV in newborn infants. ${ }^{16}$ If the infant's lungs are relatively compliant the ventilator pressure will be transmitted to the circulation thus producing a resistance to venous return to the right atrium, which would tend to reduce cardiac output. In addition, increased jugular venous pressure constitutes a resistance to cerebral blood flow.

In conclusion, the cerebral circulation of the hypotensive newborn piglet does not vasoconstrict in response to hypocapnia but positive pressure hyperventilation may significantly impair the cerebral circulation and oxygenation. Thus it would seem wise to avoid hypocapnia in mechanically ventilated infants particularly those with hypotension, hypovolaemia, or anaemia.

AW was supported by the Laerdal Foundation for Acute Medicine. MT, BRK, and KH were supported by the Norwegian Council for Science and the Humanities. ID was supported by grant No 5234 from the Swedish Medical Research Council. We are grateful to Professor Hugo Lagercrantz for his cooperation.

1 Hagberg B, Hagberg G, Olow I, Von Wendt L. The changing panorama of cerebral palsy in Sweden V. The birth year panorama of cerebral palsy in Sweden 197 . Acta Paediatr Scand 1989;78:283-90.

2 Riikonen R, Raumavirta S, Sinivuori E, Seppälä T. Changing pattern of cerebral palsy in the southwest region of Finland. pattern of cerebral palsy in the south

3 Trounce JO, Shaw DE, Levene MI, Rutter N. Clinical risk factors and periventricular leucomis factors and perive

4 Greisen G, Munck H, Lou $H$. Severe hypocarbia in preterm infants and neurodevelopmental defect. Acta Paediat Scand 1987;76:401-4.

5 Harper AM, Glass HI. The effects of alterations in the arterial carbon dioxide tension on the blood flow through the cerebral cortex at normal and low arterial blood pressures. f Neurol Neurosurg Psychiatry 1965;28:449-52.

6 Cohen PJ, Reivitch M, Greenbaum L. The electroencephalogram of awake man during hyperventilation: effects of oxygen at three atmospheres (absolute) pressure. Anesthesiology 1966;27:211-2. 
7 Calvert SA, Hoskins EM, Fong KW, Forsyth SC. Etiological factors associated with the development of periventrocular leukomalacia. Acta Paediatr Scand 1987;76:254-9.

8 Nevin M, Colchester ACF, Adams S, Pepper JR. Evidence for involvement of hypocapnia and hypoperfusion in aetiology of neurological deficit after cardiopulmonary bypass. Lancet 1987;ii:1493-5.

9 Irestedt L, Dahlin I, Hertzberg T, Sollevi A, Lagercrantz H. Adenosine concentration in umbilical cord blood of newborn infants after vaginal delivery and cesarean section. newborn infants after vaginal
Pediatr Res 1989;26:106-8.

10 Noetter GE. Introduction to statistics. Boston: Houghton Mifflin, 1971:106-19.

11 Guldvog I, Kjærnes M, Thoresen M, Walløe L. Blood flow in arteries determined transcutaneously by an ultrasonic Doppler velocimeter as compared to electromagnetic measurements on the exposed vessels. Acta Physiol Scand
1980;109:211-6.

12 Saugstad $\mathrm{O}$. Hypoxanthine as an indicator of hypoxia: its role in health and disease through free radical production. Pediatr Res 1988;23:143-9.

13 Granholm L, Siesjo BK. The effect of combined respiratory and nonrespiratory alkalosis on energy metabolism and acid-base parameters in the rat brain. Acta Physiol Scand 1971;81:307-14.

14 Nilsson L, Busto R. Controlled hyperventilation and its effect on brain energy and acid-base parameters. Acta Anaesthesiol

15 Mitchenfelder JD, Theye RA. The effects of profound hypocapnia and dilutional anemia on canine cerebral metabolism and blood flow. Anesthesio

16 Cowan $F$, Thoresen $M$. The effects of intermittent positive pressure on the cerebral arterial and venous blood flow in the newborn infant. Acta Paediatr Scand 1987;76:239-47. 